7. Loftis AJ, Quellie S, Chason K, Sumo E, Toukolon M, Otieno Y, et al. Validation of the Cepheid GeneXpert for detecting Ebola virus in semen. J Infect Dis. 2017;215:344-50.

8. Pettitt J, Higgs E, Fallah M, Nason M, Stavale E, Marchand J, et al. Assessment and optimization of the GeneXpert diagnostic platform for detection of Ebola virus RNA in seminal fluid. J Infect Dis. 2017;215:547-53.

Address for correspondence: Lisa E. Hensley, Integrated Research Facility at Fort Detrick, Division of Clinical Research, National Institute of Allergy and Infectious Diseases, National, Institutes of Health, B-8200 Research Plaza, Fort Detrick, Frederick, MD 21702, USA; email: lisa.hensley@nih.gov

\section{Polyresistant Mycobacterium bovis Infection in Human and Sympatric Sheep, Spain, 2017-2018}

\author{
Bernat Pérez de Val, Beatriz Romero, \\ María Teresa Tórtola, Laura Herrera León, \\ Pilar Pozo, Irene Mercader, Jose Luís Sáez, \\ Mariano Domingo, Enric Vidal
}

\begin{abstract}
Author affiliations: Institut de Recerca i Tecnología
Agroalimentàries-Centre de Recerca en Sanitat Animal, Barcelona, Spain (B. Pérez de Val, M. Domingo, E. Vidal); VISAVET-Universidad Complutense de Madrid, Madrid, Spain (B. Romero, P. Pozo); Vall d'Hebron Research Institute, Barcelona (M.T. Tórtola); Instituto de Salud Carlos III, Majadahonda, Madrid (L.H. León); MAEVA SERVET, S.L., Madrid (P. Pozo); Department d'Agricultura, Ramaderia, Pesca i Alimentació de la Generalitat de Catalunya, Barcelona (I. Mercader); Ministerio de Agricultura, Pesca y Alimentación, Madrid (J.L. Sáez); Universitat Autònoma de Barcelona, Barcelona (M. Domingo)
\end{abstract}

DOI: https://doi.org/10.3201/eid2704.204467

The main etiologic agent of tuberculosis (TB) in livestock is Mycobacterium bovis; human TB cases caused by $M$. bovis are rare. Analysis of a TB outbreak caused by polyresistant $M$. bovis involving a human and sympatric sheep in Spain suggests local circulation of drug-resistant $M$. bovis strains among livestock.
The main etiologic agent of tuberculosis (TB) in 1 livestock and wildlife is Mycobacterium bovis. This species also infects humans through inhalation or ingestion and causes TB that is clinically indistinguishable from that caused by $M$. tuberculosis.

In 2017, a case of pulmonary TB caused by $M$. bovis in a human was detected in the Vall d'Hebron Hospital in Barcelona, Spain. Bacteriological culture of clinical specimens in Löwenstein-Jensen and 7H9 media (BD Diagnostics, https://bd.com), followed by antimicrobial resistance testing (BACTEC MGIT 960; BD Diagnostics), revealed a strain resistant to 2 first-line anti-TB drugs: pyrazinamide $(100 \mu \mathrm{g} /$ $\mathrm{mL})$ and isoniazid $(0.1 \mu \mathrm{g} / \mathrm{mL})$. A complementary analysis, performed by using the proportion method, confirmed resistance to isoniazid $(0.2 \mu \mathrm{g} / \mathrm{mL})$, elucidating a polyresistant case of TB (resistance to $>1$ first-line anti-TB drug other than both isoniazid and rifampin); the strain was also resistant to ethionamide $(30 \mu \mathrm{g} / \mathrm{mL})$, an antimicrobial drug specifically used to treat active multidrug-resistant TB (resistance to at least both isoniazid and rifampicin). Molecular characterization by direct variable repeat (DVR)-spoligotyping identified the isolates as M. bovis spoligopattern SB0124 (http:/ / www.mbovis.org).

The patient worked as a farmer on cattle and small ruminant farms in his county. Therefore, the epidemiologic investigation included the livestock he was in contact with, particularly the herd of sheep and goats he was currently managing. In 2018, a total of $34(25 \%)$ ewes and $3(18 \%)$ goats had positive results to a single intradermal tuberculin test, interferon gamma release assay (IDvet, https://www.idvet.com), or both. Animals with positive test results were slaughtered, and tissues from 23 (21 sheep and 2 goats) were examined postmortem. TB-compatible lesions were found in the lungs and thoracic, mesenteric, or ileocecal lymph nodes of 13 animals (12 sheep, 1 goat). Tissues with lesions were cultured in Löwenstein-Jensen with pyruvate and Coletsos and in 7H9 media by using BACTEC MGIT 320 (all BD Diagnostics). Culture indicated growth of $M$. tuberculosis complex in 9 sheep samples, and M. avium subspecies avium was isolated from another sheep and the goat. DVR-spoligotyping was performed for the $9 \mathrm{M}$. tuberculosis complex isolates, and M. bovis SB0124 was identified in all. This unusual spoligopattern had also been identified in a cattle herd in the same county in 2005 (Spanish Database of Animal Mycobacteriosis; https://www.visavet.es/mycodb); the patient had no known connection to that herd.

Genome sequence analysis based on assessment of single-nucleotide polymorphisms (SNPs) 
was conducted for 2 isolates from sheep (2018) and the isolate from the human patient (2017) and for 2 isolates collected from cattle in 2005-2006. Results showed an extremely close phylogenetic relationship between the isolates from the sheep and human ( $<5$ SNPs), leading us to conclude that they were the same strain; they differed from the strains from cattle by 35-38 SNPs (Figure). Of note, the isolates from the sheep and human showed resistance to pyrazinamide, isoniazid, and ethionamide, and isolates from the cattle showed resistance to pyrazinamide and isoniazid. These results suggest that although strains from cattle and from the sheep and human were not closely related enough to be considered the same strain, they might have evolved from a common ancestral isoniazid-resistant strain. However, mutations associated with pyrazinamide resistance were found only at the pncA (C169G) gene and with isoniazid/ethionamide resistance at the inh $\mathrm{A}$ (T280G) gene, although the inhA modification was detected only in the isolates from cattle.

Human TB caused by M. bovis is usually associated with occupational exposure and is infrequently reported in Spain; cases of multidrug-resistant (MDR) TB are even more rare $(1,2)$. However, zoonotic cases could be underestimated because the need for relatively sophisticated laboratory methods hinders estimation of zoonotic TB occurrence, particularly in low-income areas, and epidemiologic relationships between TB patients and sympatric livestock are rarely investigated.
Although only a few cases of TB in sheep have been reported in Spain $(3,4)$, these reports suggest that sheep can play a role as maintenance hosts of $M$. bovis and M. caprae in certain epidemiologic situations. TB progression in sheep appears to be similar to that in cattle or goats (5).

M. bovis is naturally resistant to pyrazinamide (6), but our findings reveal circulation of polyresistant strains in livestock in the outbreak area. In contrast, a previous study reported absence of polyresistant $M$. bovis strains isolated from livestock in the Iberian Peninsula (7). Similarly, cases of TB in humans caused by isoniazid-resistant $M$. bovis are infrequent in Spain (8), although a nosocomial outbreak caused by MDR M. bovis involving HIV-infected patients has been described (9). Only a few studies have examined treatment of isoniazid polydrug resistance (10), which is particularly dangerous because of the high risk that resistance to rifampin will develop, requiring full MDR TB treatment.

Distinguishing between TB causative organisms is crucial for epidemiologic investigation and adequate treatment of TB in humans. The One Health approach should be implemented in contact investigations for TB cases through coordination of public and animal health authorities to prevent spread of TB between humans and livestock. Controlling TB in small ruminants and studying drug resistance in strains circulating among livestock should also be considered.

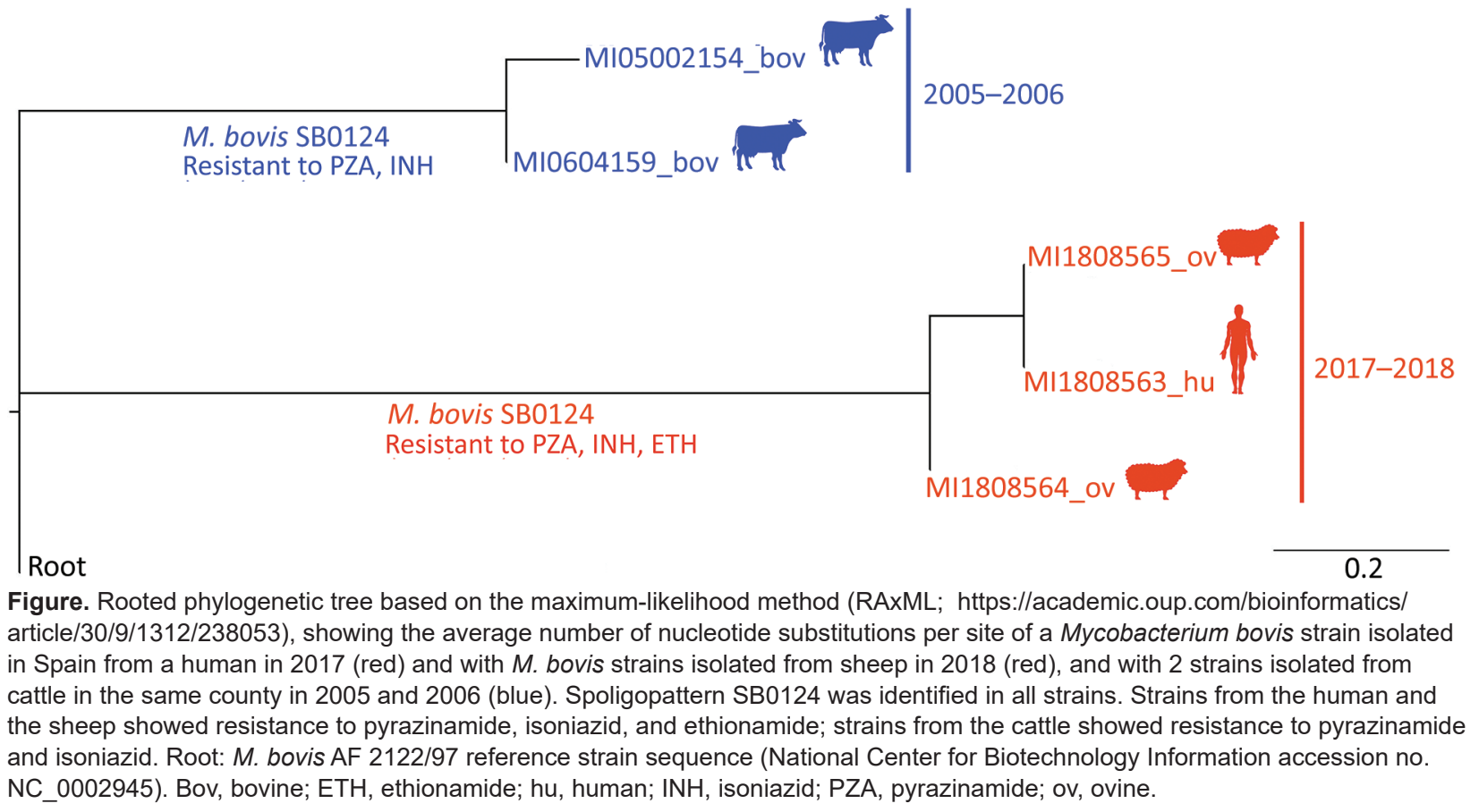




\section{Acknowledgments}

We are grateful to Maite Martín and Mónica Pérez for their outstanding technical assistance and to the Mycobacteria Section of the National Veterinary Services Laboratories (US Department of Agriculture, Animal and Plant Health Inspection Service) for the sequencing service and technical support.

This work was supported by the Department of Agriculture, Livestock Fisheries and Food of the Government of Catalonia and by the Spanish Ministry of Agriculture, Fisheries and Food. The Institute of Agrifood Research and Technology is supported by Centres de Recerca de Catalunya Programme/Generalitat de Catalunya.

\section{About the Author}

Dr. Pérez de Val is a researcher at the Institut de Recerca i Tecnología Agroalimentàries-Centre de Recerca en Sanitat Animal, Barcelona, Spain, where he conducts research on control and surveillance of TB.

\section{References}

1. Samper S, Iglesias MJ, Rabanaque MJ, Gómez LI, Lafoz MC, Jiménez MS, et al.; Spanish Working Group on MDR-TB. Systematic molecular characterization of multidrug-resistant Mycobacterium tuberculosis complex isolates from Spain. J Clin Microbiol. 2005;43:1220-7. https://doi.org/10.1128/ JCM.43.3.1220-1227.2005

2. Rodríguez E, Sánchez LP, Pérez S, Herrera L, Jiménez MS, Samper S, et al. Human tuberculosis due to Mycobacterium bovis and M. caprae in Spain, 2004-2007. Int J Tuberc Lung Dis. 2009;13:1536-41.

3. Muñoz Mendoza M, Juan L, Menéndez S, Ocampo A, Mourelo J, Sáez JL, et al. Tuberculosis due to Mycobacterium bovis and Mycobacterium caprae in sheep. Vet J. 2012;191:2679. https://doi.org/10.1016/j.tvj1.2011.05.006

4. Vidal E, Grasa M, Perálvarez T, Martín M, Mercader I, Pérez de Val B. Transmission of tuberculosis caused by Mycobacterium caprae between dairy sheep and goats. Small Rumin Res. 2018;158:22-5. https:// doi.org/10.1016/ j.smallrumres.2017.11.010

5. Balseiro A, Altuzarra R, Vidal E, Moll X, Espada Y, Sevilla IA, et al. Assessment of BCG and inactivated Mycobacterium bovis vaccines in an experimental tuberculosis infection model in sheep. PLoS One. 2017;12:e0180546. https://doi.org/10.1371/journal. pone. 0180546

6. Scorpio A, Collins D, Whipple D, Cave D, Bates J, Zhang Y. Rapid differentiation of bovine and human tubercle bacilli based on a characteristic mutation in the bovine pyrazinamidase gene. J Clin Microbiol. 1997;35:106-10. https://doi.org/10.1128/JCM.35.1.106-110.1997

7. Romero B, Aranaz A, Bezos J, Alvarez J, de Juan L, Tariq Javed M, et al. Drug susceptibility of Spanish Mycobacterium tuberculosis complex isolates from animals. Tuberculosis (Edinb). 2007;87:565-71. https:// doi.org/ 10.1016/j.tube.2007.08.004

8. Nebreda-Mayoral T, Brezmes-Valdivieso MF, Gutiérrez-Zufiaurre N, García-de Cruz S, Labayru-Echeverría C, López-Medrano R, et al. Human
Mycobacterium bovis infection in Castile and León (Spain), 2006-2015 [in Spanish]. Enferm Infecc Microbiol Clin. 2019;37:19-24. https:// doi.org/10.1016/j.eimc.2017.11.018

9. Samper S, Martín C, Pinedo A, Rivero A, Blázquez J, Baquero F, et al. Transmission between HIV-infected patients of multidrug-resistant tuberculosis caused by Mycobacterium bovis. AIDS. 1997;11:1237-42. https://doi.org/10.1097/00002030-199710000-00006

10. Menzies D, Benedetti A, Paydar A, Royce S, Madhukar P, Burman W, et al. Standardized treatment of active tuberculosis in patients with previous treatment and/or with mono-resistance to isoniazid: a systematic review and meta-analysis. PLoS Med. 2009;6:e1000150. https:/ / doi.org/ 10.1371/journal.pmed.1000150

Address for correspondence: Bernat Pérez de Val, Institut de Recerca i Tecnologia Agroalimentaries Centre de Recera en Sanitat Animal Edifici CReSA. Campus of the Autonomous University of Barcelona, 08193-Bellaterra, Barcelona, Spain; email: bernat.perez@irta.cat

\section{Novel SARS-CoV-2 Variant in Travelers from Brazil to Japan}

\author{
Takahisa Fujino, Hidetoshi Nomoto, \\ Satoshi Kutsuna, Mugen Ujiie, Tetsuya Suzuki, \\ Rubuna Sato, Tsuguto Fujimoto, Makoto Kuroda, \\ Takaji Wakita, Norio Ohmagari
}

Author affiliations: National Center for Global Health and Medicine, Tokyo, Japan (T. Fujino, H. Nomoto, S. Kutsuna, M. Ujiie, T. Suzuki, R. Sato, N. Ohmagari); Tohoku University, Miyagi, Japan (H. Nomoto, T. Suzuki, N. Ohmagari); National Institute of Infectious Diseases, Tokyo (T. Fujimoto, M. Kuroda, T. Wakita)

\section{DOI: https://doi.org/10.3201/eid2704.210138}

Multiple severe acute respiratory syndrome coronavirus 2 (SARS-CoV-2) variants with higher transmission potential have been emerging globally, including SARS-CoV-2 variants from the United Kingdom and South Africa. We report 4 travelers from Brazil to Japan in January 2021 infected with a novel SARS-CoV-2 variant with an additional set of mutations. 